\title{
Graphene-Based Electron Transport Layers in Perovskite Solar Cells: A Step-Up for an Efficient Carrier Collection
}

\author{
Francesco Biccari, Fabio Gabelloni, Erica Burzi, Massimo Gurioli, Sara Pescetelli, \\ Antonio Agresti, Antonio Esaú Del Rio Castillo, Alberto Ansaldo, Emmanuel Kymakis, \\ Francesco Bonaccorso, Aldo Di Carlo, and Anna Vinattieri*
}

The electron transport layer (ETL) plays a fundamental role in perovskite solar cells. Recently, graphene-based ETLs have been proved to be good candidate for scalable fabrication processes and to achieve higher carrier injection with respect to most commonly used ETLs. Here, the effects of different graphenebased ETLs in sensitized methylammonium lead iodide (MAPI) solar cells are experimentally studied. By means of time-integrated and picosecond time-resolved photoluminescence techniques, the carrier recombination dynamics in MAPI films embedded in different ETLs is investigated. Using graphene doped mesoporous $\left.\mathrm{TiO}_{2}(\mathrm{G}+\mathrm{mTiO})_{2}\right)$ with the addition of a lithiumneutralized graphene oxide (GO-Li) interlayer as ETL, it is found find that the carrier collection efficiency is increased by about a factor two with respect to standard $\mathrm{mTiO}_{2}$. Taking advantage of the absorption coefficient dispersion, the MAPI layer morphology is probed, along the thickness, finding that the MAPI embedded in the ETL composed by $\mathrm{G}+\mathrm{mTiO}_{2}$ plus $\mathrm{CO}$-Li brings to a very good crystalline quality of the MAPI layer with a trap density about one order of magnitude lower than that found with the other ETLs. In addition, this ETL freezes MAPI at the tetragonal phase, regardless of the temperature. Graphene-based ETLs can open the way to significant improvement of perovskite solar cells.

\section{Introduction}

In the last decade, hybrid organicinorganic materials with the perovskite structure, most notably $\mathrm{CH}_{3} \mathrm{NH}_{3} \mathrm{~Pb}(\mathrm{I}, \mathrm{Cl})_{3}$, have become the most promising lightharvesting active layer for the implementation of high efficiency and low cost solar cells. ${ }^{[1-8]}$ As a matter of fact, the power conversion efficiency of perovskite solar cells (PSCs) has shown a very fast growth, increasing from 3.8\% ${ }^{[9]}$ in 2009 to $22.1 \%^{[10]}$ in 2016 . The ease of fabrication and the high radiative efficiency have made perovskites also extremely attractive for the development of bright LEDs and lasers. ${ }^{[11-14]}$

Nevertheless, a major drawback originates from the poor material stability. ${ }^{[3,15]}$ decomposition after exposure to moisture, cracks and defects generated by thermal stresses, crystal phase transition, UV-light exposure represent the principal causes of aging. ${ }^{[3,15]}$ Moreover, a high density of trap states, both on surfaces and in grain boundaries, ${ }^{[3,16-18]}$ are present in poly-

Dr. F. Biccari, Dr. F. Gabelloni, Dr. E. Burzi, Prof. M. Gurioli,

Prof. A. Vinattieri

Department of Physics and Astronomy

University of Florence and LENS

Via Sansone 1, I-50019 Sesto Fiorentino (FI), Italy

E-mail: anna.vinattieri@unifi.it

Dr. S. Pescetelli, Dr. A. Agresti, Prof. A. Di Carlo

C.H.O.S.E. (Centre for Hybrid and Organic Solar Energy)

Department of Electronic Engineering

University of Rome Tor Vergata

Via del Politecnico 1, I-00133 Rome, Italy

Dr. A. E. Del Rio Castillo, Dr. A. Ansaldo, Dr. F. Bonaccorso

Istituto Italiano di Tecnologia

Graphene Labs

Via Morego 30, I-16163 Genova, Italy

Prof. E. Kymakis

Center of Materials Technology and Photonics \& Electrical Engineering

Department, School of Applied Technology

Technological Educational Institute (T.E.I) of Crete, Heraklion

71004 Crete, Greece

The ORCID identification number(s) for the author(s) of this article can be found under https://doi.org/10.1002/aenm.201701349.

DOI: 10.1002/aenm.201701349 crystalline perovskites, used for solar cells and light emitters. Indeed, even though theoretical predictions show that deep trap states are not generally formed inside perovskites grains, the opposite is observed at the grain boundaries and at the surfaces. ${ }^{[3,19]}$ Therefore, sophisticated passivation strategies are essential for increasing the efficiency of PSCs and light emitters. ${ }^{[19-22]}$

For $\mathrm{CH}_{3} \mathrm{NH}_{3} \mathrm{PbI}_{3}$ (methylammonium lead iodide, MAPI) perovskites, a correlation between the solar cell efficiency and the grain morphology was recently demonstrated. ${ }^{[23]}$ Results of local short-circuit photocurrent, open-circuit voltage, and dark drift current within individual grains correlate these quantities to different crystal facets, as a consequence of a facet-dependent density of trap states ${ }^{[24]}$ and it was also proven that the structural order of the electron transport layer (ETL) impacts the overall cell performance. ${ }^{[23]}$ Moreover, the nature of grain boundaries was shown to affect the carrier recombination kinetics because of nonradiative pathways that would also play a role in the process of charge separation and collection. ${ }^{[22]}$

It turns out that the possibility of controlling the morphology of the perovskite thin film and the understanding if different 
realizations of the ETL can modify the morphology of the grains are of the utmost relevance to further improvements of perovskite based solar cells.

Recently, graphene and related 2D materials have been introduced in the device structure in order to improve the charge injection and/or collection at the electrodes: an enhancement of the power conversion efficiency ${ }^{[25]}$ and a long-term stability ${ }^{[26]}$ was obtained. As a matter of fact, interfaces between perovskite and transport layers have been recently demonstrated to dramatically affect the charge recombination processes and material instability within the working device. ${ }^{[27]}$ In fact, when free charges are fast injected from perovskite to the electron transport layer, the perovskite degradation is slowed down and the nonradiative recombination is reduced. ${ }^{[28]}$ In particular, the insertion of graphene flakes into the mesoporous- $\mathrm{TiO}_{2}$ layer $\left(\mathrm{mTiO}_{2}\right)$ and of lithium-neutralized graphene oxide (GO-Li) as interlayer at perovskite $/ \mathrm{mTiO}_{2}$ interface showed enhanced conversion efficiency and stability on both small and large area devices by demonstrating the crucial role of graphene interface engineering in perovskite-based devices. ${ }^{[2]}$ Thus, the influence of mesoscopic-graphene modified substrates onto the perovskite film need to be investigated more in details to finely control the photovoltaic performance of complete devices.

Given the typical size of the grains (a few hundreds of nanometers), high resolution techniques as transmission electron microscopy (TEM), atomic-force microscopy (AFM), scanning near-field optical microscopy (SNOM), etc. are employed to investigate the grain morphology but a difficult task is to correlate physical properties at the nanoscale with the device performance measuring, for instance, the $I-V$ curve of the cell. ${ }^{[24]}$ Thus, it would be of extreme relevance to extract information on the active film morphology with much easier techniques on a length scale of tens/hundreds of micrometers and even larger so to assess the homogeneity of the film deposition and the reliability of the synthesis protocol and postdeposition treatments. Moreover, the high spatial resolution analysis can explore a limited thickness of the film, and therefore it appears difficult to get information in the case of a real device. Photoluminescence (PL) spectroscopy is an effective tool to investigate the film quality: in fact, from the comparison of samples with different ETLs, it is possible to extract quantitative information on the carrier capture and transport and, by the spectral shape, identify the crystalline phase of the active layer and evaluate the density of traps/defects. Moreover, given the limited thickness (few hundreds of $\mathrm{nm}$ ) of the perovskite film in solar cells and given the steep behavior of the absorption coefficient in MAPI, ${ }^{[30]}$ by varying the excitation wavelength in the range $300-700 \mathrm{~nm}$, it is possible to probe thicknesses of the film from a hundred of $\mathrm{nm}$ to the whole film layer.

In this paper we aim to establish the effects of different graphene-based ETLs in sensitized MAPI solar cells. In particular we will study the ETL effects on the carrier collection efficiency and on the MAPI morphology along the thickness. By picosecond time-resolved measurements we correlate the carrier recombination dynamics to the crystalline quality of the active material in presence/absence of the ETL. We will find an increase of the electron collection efficiency up to a factor 3 with respect to standard $\mathrm{mTiO}_{2}$. Taking advantage of the absorption coefficient dispersion, we are able to assess the film morphology along the thickness. In fact, by tuning the excitation wavelength, we investigate a thickness range from 150 to $400 \mathrm{~nm}$ and we can highlight the morphology changes induced by different ETLs. Our results will indicate that, when a graphene doped mesoporous $\mathrm{TiO}_{2}\left(\mathrm{G}+\mathrm{mTiO}_{2}\right)$ with the addition of a GO-Li interlayer is used as ETL, the morphology of the MAPI film embedded in the mesoporous layer is frozen in the tetragonal phase, regardless of the temperature. In addition, the defect concentration is about one order of magnitude lower than that found with the other ETLs.

\section{Results and Discussion}

Four types of samples were prepared using different combinations of ETLs: $\mathrm{mTiO}_{2}, \mathrm{G}+\mathrm{mTiO}_{2}, \mathrm{mTiO}_{2}$ plus GO-Li interlayer, and $\mathrm{G}+\mathrm{mTiO}_{2}$ plus GO-Li interlayer. The different sample structures are schematically shown in Figure 1 and listed in Table 1. For clear comparison a sample of MAPI on fluorine-doped tin oxide (FTO) without ETL (reference sample) was also investigated. Details on the sample preparation are reported in the Experimental Section. It is worth noting that PL analysis was carried out on simple photoelectrodes, lacking of the hole collecting layer and the bottom contact: this allows us to focus only on the electron collection and transport after the electron-hole pairs creation due to the photon absorption. Moreover, PL experiments can be realized illuminating the samples either from the perovskite film side (side A) or the FTO side (side B). Varying the excitation wavelength and the excitation side, we can differently penetrate into the MAPI film and selectively probe spatial regions few tens of $\mathrm{nm}$ near the ETL or far from it.

In Figure 2 PL decays at room temperature, after excitation with photons of $2.06 \mathrm{eV}$, are compared for the different samples, while in the inset a PL spectrum at room temperature is shown: typical spectra of the tetragonal phase are observed. ${ }^{[31,32]}$ By exciting from side A (Figure 2a), the PL decays are identical for all samples and no effect related to the presence of the ETL is detected. In contrast there is a significant difference in the

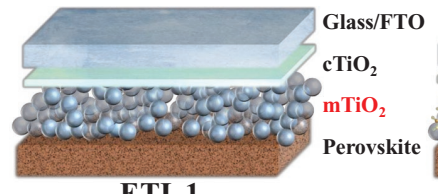
ETL 1

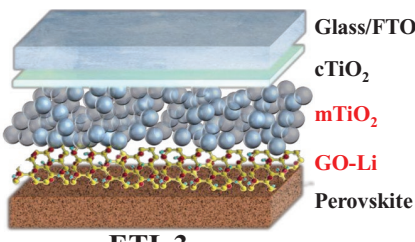

ETL 3

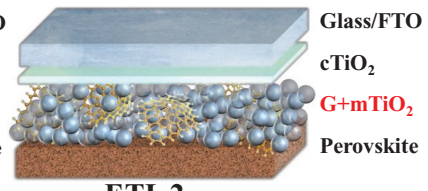
ETL 2

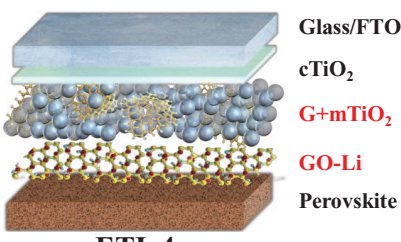

ETL 4

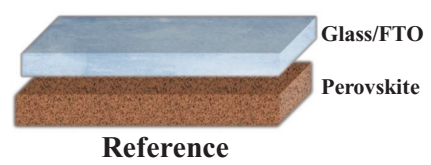

Figure 1. Structures of the investigated samples. The ETLs are indicated in red. 
Table 1. List of the investigated samples and description of their corresponding ETLs.

\begin{tabular}{lc}
\hline Sample & ETL \\
\hline Reference & $\mathrm{No} \mathrm{ETL}$ \\
ETL 1 & $\mathrm{mTiO}_{2}$ \\
ETL 2 & $\mathrm{G}+\mathrm{mTiO}_{2}$ \\
ETL 3 & $\mathrm{mTiO}_{2}$ plus GO-Li \\
ETL 4 & ${\mathrm{G}+\mathrm{mTiO}_{2} \text { plus GO-Li }}$ \\
\hline
\end{tabular}

PL decay by exciting from side B depending on the sample, as shown in Figure $2 \mathrm{~b}$ : faster decays are observed in presence of ETL, especially when graphene and/or GO-Li are used. It is worth noting that the PL decay of reference sample excited from side B is slower with respect to the decays of side A. This can be attributed to nonradiative states at the surface of the uncovered perovskite film (side A).

To extract information about the efficiency in the carrier injection from perovskite to ETL, we first fitted the decays of Figure $2 \mathrm{~b}$ with a double exponential, ${ }^{[20,33]}$ taking into account the laser pulse repetition period. ${ }^{[34]}$ The fitting function $I(t)$ can be written as

$I(t)=f(t)+g(t)$

where $f(t)$ is the original double exponential and $g(t)$ is the correction term due to the intrinsic periodic nature of time-correlated single photon counting technique (TCSCP) measurements. ${ }^{[34]}$

$$
\begin{aligned}
& f(t)=\theta\left(t-t_{0}\right)\left[C \mathrm{e}^{-\left(t-t_{0}\right) / \tau_{1}}+(1-C) \mathrm{e}^{-\left(t-t_{0}\right) / \tau_{2}}\right] \\
& g(t)=C \frac{\mathrm{e}^{-\left(t-t_{0}\right) / \tau_{1}}}{\mathrm{e}^{T / \tau_{1}}-1}+(1-C) \frac{\mathrm{e}^{-\left(t-t_{0}\right) / \tau_{2}}}{\mathrm{e}^{T / \tau_{2}}-1}
\end{aligned}
$$

In the previous equations $\theta(t)$ is the Heaviside step function, $\tau_{1}$ and $\tau_{2}$ are the decay time constants, $T$ is the laser pulse
Table 2. Results of the fits of data of Figure $2 \mathrm{~b}: \tau_{1}$ and $\tau_{2}$ are the decay time constants, $C$ is the contribution of $\tau_{1}$ to the fit and $\eta$ is the radiative efficiency.

\begin{tabular}{lcccc}
\hline Sample & $\tau_{1}[\mathrm{~ns}]$ & $\tau_{2}[\mathrm{~ns}]$ & $C$ & $H$ \\
\hline Reference & 25 & - & 1.00 & 1 \\
ETL 1 & 25 & 2.05 & 0.43 & 0.48 \\
ETL 2 & 15 & 1.99 & 0.36 & 0.27 \\
ETL 3 & 15 & 1.24 & 0.20 & 0.16 \\
ETL 4 & 15 & 1.30 & 0.34 & 0.24 \\
\hline
\end{tabular}

repetition period ( $13.15 \mathrm{~ns}$ ), $C$ is the contribution of $\tau_{1}$ exponential to the fit, and $t_{0}$ is a constant. The results obtained by the fitting procedure are reported in Table 2. Inserting graphenebased ETLs in the samples reduces $\tau_{1}$ from 25 to 15 ns while the reduction of $\tau_{2}$ depends on the ETL. In the literature ${ }^{[20,33]}$ the longer decay constant $\left(\tau_{1}\right)$ is ascribed to the radiative recombination in MAPI while the shorter decay constant can be attributed to the carrier removal from MAPI layer toward the ETL.

To get rid of the local inhomogeneities in the samples, which can give rise to variation of the time integrated (TI)-PL intensity and considering that the PL time evolution does not depend on the detection spot, we estimate the TI-PL intensity from the PL decay. It turns out that we can express the integrated PL intensity $\left(I_{\mathrm{PL}}\right)$ as

$$
I_{\mathrm{PL}}=\eta P
$$

where $\eta$ is the radiative efficiency and $P$ is the pump intensity, equals to $10 \mathrm{~W} \mathrm{~cm}^{-2}$ for all the time resolved (TR)-PL measurements. Assuming a unitary radiative efficiency for the reference sample, we can evaluate the change in $\eta$ for the different ETLs (see Table 2). Lower $\eta$ is obtained in case of ETLs 2, 3, and 4: thus the insertion of graphene and/or GO-Li interlayer improves the electron capture from perovskite to ETL by a factor between two and three with respect to ETL 1 . We want to remark that this result does not necessarily imply an increase
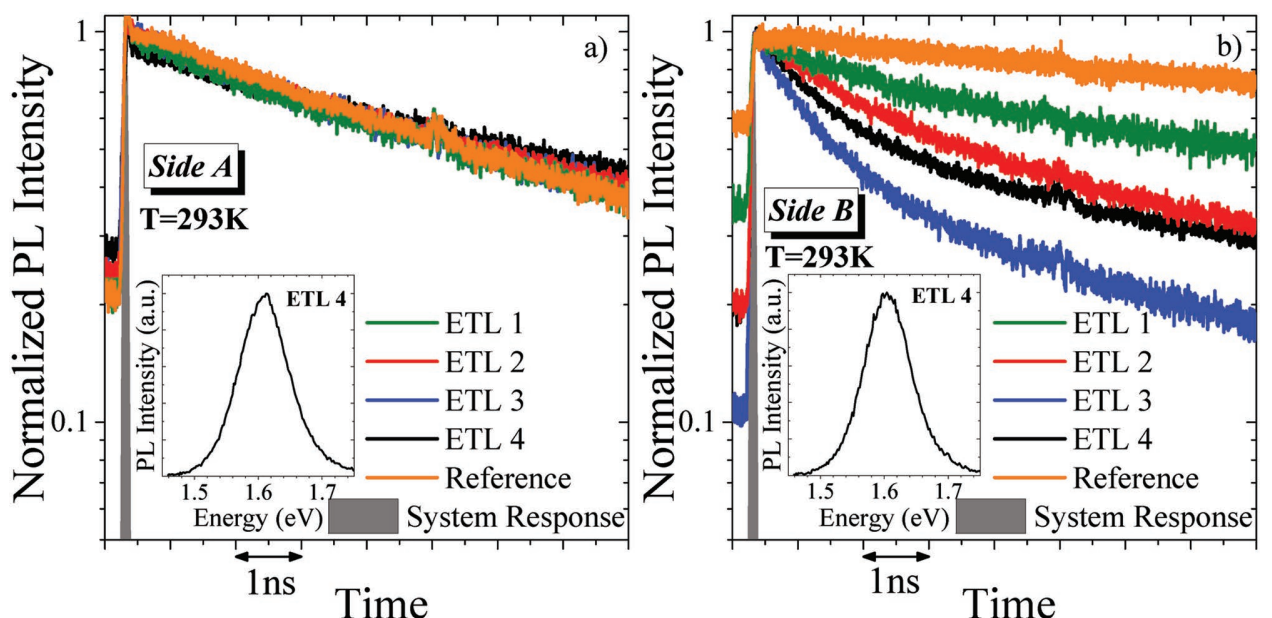

Figure 2. PL decay (at the PL peak energy) and PL spectra (in the inset) at room temperature, after ps excitation at $2.06 \mathrm{eV}$ with an average intensity of $10 \mathrm{~W} \mathrm{~cm}^{-2}$. a) From side A. b) From side B. 
of the solar cell short circuit current density $\left(J_{\mathrm{sc}}\right)$ of the same amount. In fact hole collection by the hole transport layer ${ }^{[35]}$ and nonradiative recombination in the ETL have to be taken into account. However, as shown in the Supporting Information, a significant increase of $J_{\mathrm{sc}}$ is measured for complete cell when ETL 4 is used.

More insight in the role of the ETL can be obtained by PL spectra at low temperature ( $T=11 \mathrm{~K})$. In Figure $3 \mathrm{PL}$ spectra, obtained by exciting the samples from side A (Figure 3a) and side $\mathrm{B}$ (Figure $3 \mathrm{~b}$ ), are reported. The PL spectra from side A show two peaks. As expected for $T<150 \mathrm{~K}$ in MAPI perovskite, ${ }^{[31,36]}$ the peak at about $1.65 \mathrm{eV}$ is attributed to the orthorhombic phase of MAPI. But the major contribution to the spectra comes from the other peak, centered at $1.55 \mathrm{eV}$. This emission is likely due to the sum of two contributions: the radiative recombination arising from the residual tetragonal phase at $1.56 \mathrm{eV}$ and the radiative recombination from localized states below $1.52 \mathrm{eV}$. In the literature, these localized states are identified with radiative traps ${ }^{[17,37,38]}$ or, recently, to methylammonium-disordered domains in orthorhombic phase of MAPI ${ }^{[32]}$ We want to remark that, in both interpretations of the low energy side emission as radiative traps or disordered domains, a carrier localization is present. More relevant is the fact that the low energy states are radiative and do not produce a loss of photogenerated carriers.

Considering that the absorption length of the MAPI film at about $2 \mathrm{eV}$ is roughly $200 \mathrm{~nm}^{[30]}$ and that the thickness of the perovskite layer, for all the analyzed samples, is about $350 \mathrm{~nm}$ (see Figure S5 in the Supporting Information), we can conclude that the emission, exciting from side A, comes mostly from the MAPI film and that no effect related to the presence of the ETL is detected. On the contrary, the excitation from side B can reveal the ETL effect on the MAPI. As a matter of fact, relevant differences are observed between the emissions from side B (Figure 3b). First of all we observe a smaller contribution of the orthorhombic phase for all the samples with respect to the excitation from side A. Moreover, in the case of GO-Li plus $\mathrm{G}+\mathrm{mTiO}_{2}$ (ETL 4), we detect a strong reduction of the radiative traps at $1.52 \mathrm{eV}$ and the dominance of the emission from the tetragonal phase at $1.56 \mathrm{eV}$.

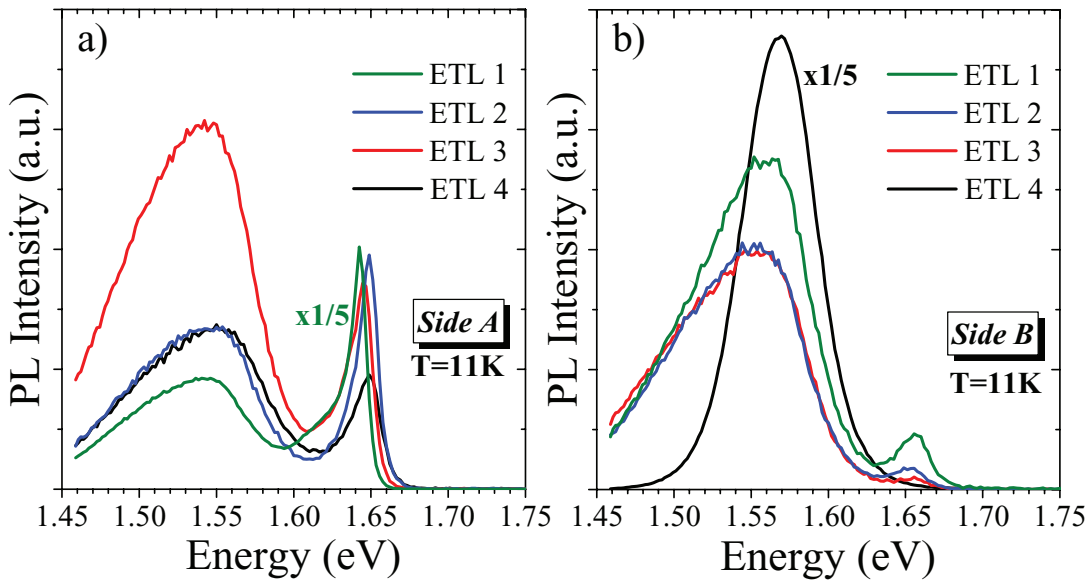

Figure 3. TI-PL spectra at $11 \mathrm{~K}$ for the various samples after excitation at $2.06 \mathrm{eV}$ with an average intensity of $10 \mathrm{~W} \mathrm{~cm}^{-2}$. a) From side A. b) From side B.
Such results suggest that an incomplete phase transition occurs for the perovskite wrapped into the mesoporous ETL layer. In particular, in the case of ETL 4, where the PL lineshape shows a negligible low energy tail and a smaller linewidth with respect to the other samples, we can argue that the crystallization of the MAPI film is very good, as also confirmed by the scanning electron microscopy (SEM) image reported in Figure S4 of the Supporting Information. However, the interaction of MAPI and ETL inhibits the phase transition, at least for a thickness of about $200 \mathrm{~nm}$ (see SEM cross-section in Figure S5 of the Supporting Information). In order to confirm and test our hypothesis we performed PL measurements as a function of temperature, excitation wavelength and excitation power.

In Figure 4a we report the emission spectra of ETL 4 exciting from side A, varying the sample temperature from 10 to $300 \mathrm{~K}$. As already shown before, at low temperature we observe two bands, one at $1.65 \mathrm{eV}$ corresponding to the orthorhombic phase and one at about $1.55 \mathrm{eV}$ corresponding to the sum of the emission from optically active trap states and the emission from a residual tetragonal phase. As expected, $[32,36,37,39]$ increasing the temperature, the orthorhombic phase emission shifts at higher energy (see Figure 4c), showing a monotonic increase of its full width at half maximum (FWHM) (see Figure 4d), and it disappears above $150 \mathrm{~K}$, where the phase transition of MAPI from orthorhombic to tetragonal phase occurs. By increasing the temperature, the low energy band shows instead the typical $\mathrm{S}$ shape both in the peak emission energy (see Figure 4c) and its FWHM (see Figure 4d). This is an indication of the phase transition from orthorhombic to tetragonal phase, with the concurrent lower contribution of the traps. Above $150 \mathrm{~K}$ the PL spectrum has only one peak, arising from the tetragonal phase emission, which continues to monotonically blue shift increasing the temperature (see Figure 4c), as expected. ${ }^{[32,36,37,39]}$ Apart from different relative weights of the emission bands, for all samples we find PL spectra very similar to the one of the sample with ETL 4 when the excitation is performed from side A.

A very similar trend with temperature, as shown in Figure 4a for ETL 4, is found for ETL 1, 2, and 3, irrespective of the excitation side, and this trend is commonly reported in the literature. ${ }^{[32]}$ On the contrary, relevant differences are observed in case of ETL 4 exciting from side B (Figure 4b). By increasing the temperature, the PL spectrum shows a single band, corresponding to the tetragonal phase, with a monotonic increase of the emission energy (see Figure $4 \mathrm{c}$ ) and the FWHM (see Figure 4d). Such behavior indicates that the MAPI film embedded in the mesoporous ETL side remains in the tetragonal phase even down to $10 \mathrm{~K}$.

The FWHM of the PL bands as a function of temperature can be fitted taking into account the temperature-independent inhomogeneous broadening and the interaction between carriers and acoustic and longitudinal optical (LO) phonons, ${ }^{[32,39]}$ using the following equation 

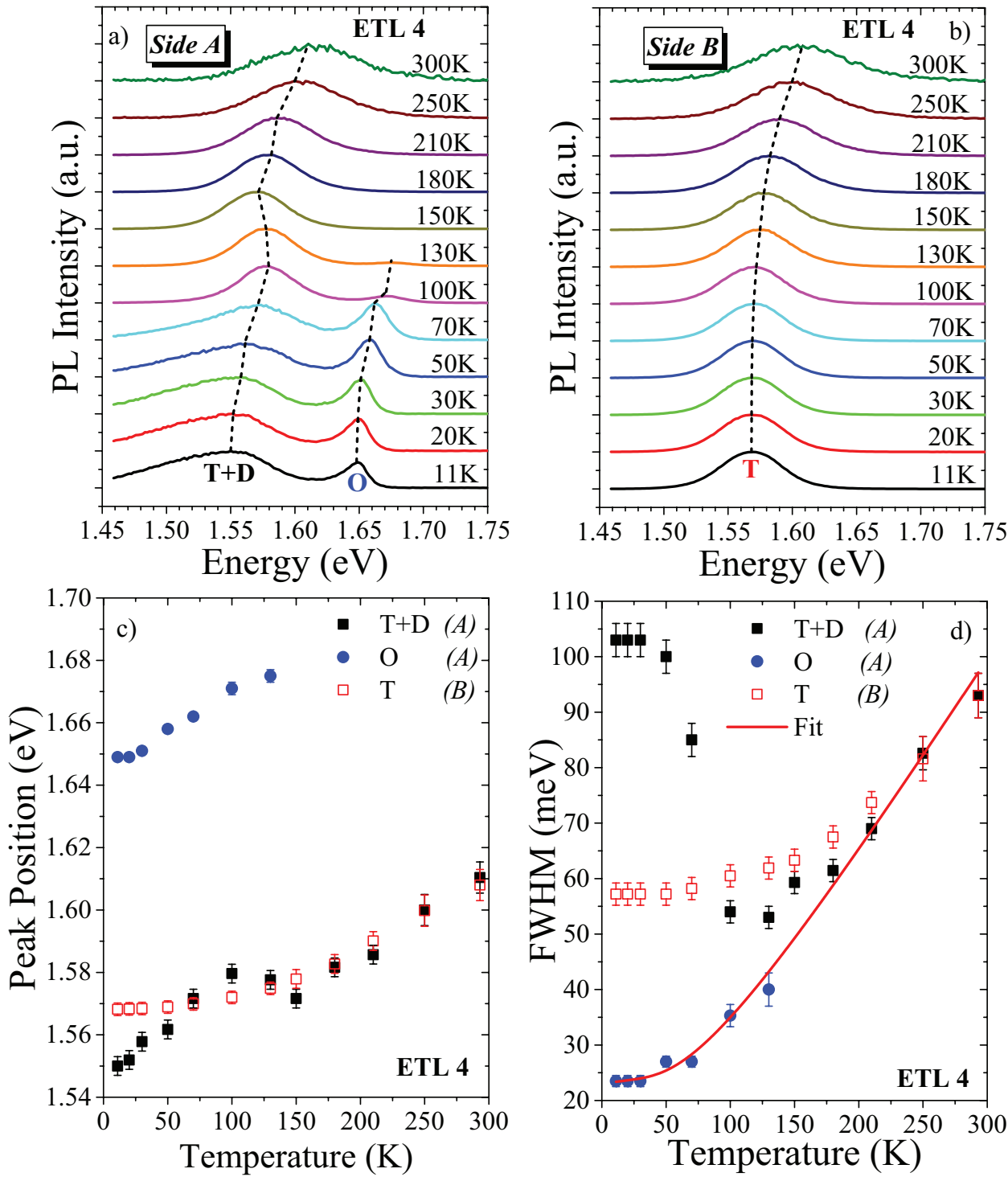

Figure 4. Temperature-dependent measurement on ETL 4 by exciting with photon energy of $2.06 \mathrm{eV}$ with an average intensity of $10 \mathrm{~W} \mathrm{~cm}^{-2}$. The labels $\mathrm{D}, \mathrm{T}$, and $\mathrm{O}$ stand for defects (trap states), tetragonal, and orthorhombic, respectively. a) PL spectra from 10 to $300 \mathrm{~K}$ after excitation on side $\mathrm{A}$. b) PL spectra from 10 to $300 \mathrm{~K}$ after excitation on side B. c) Position of the PL peaks of the spectra in (a) and (b) as a function of temperature. The excitation side is indicated in brackets. d) FWHM of the PL peaks of (a) and (b) as a function of temperature. The excitation side is indicated in brackets. Red solid line shows the fitting of FWHM through Equation (5).

$\Gamma(T)=\Gamma_{0}+\gamma_{\mathrm{ac}} T+\frac{\gamma_{\mathrm{LO}}}{\mathrm{e}^{E_{\mathrm{LO}} / k_{\mathrm{B}} T}-1}$

where $\Gamma_{0}$ is the inhomogeneous broadening, $\gamma_{\mathrm{ac}}$ and $\gamma_{\mathrm{LO}}$ are the acoustic and LO phonon-carrier coupling strengths, respectively, and $E_{\mathrm{LO}}$ is the LO phonon energy. We fitted the FWHM data extracted from the PL from side A: in particular we considered the orthorhombic phase from 10 to $150 \mathrm{~K}$ and the tetragonal phase from $150 \mathrm{~K}$ up to room temperature. If the two set of data can be fitted with a single function, we can conclude that the acoustic and optical phonons causing the PL line broadening have very similar energies for the two phases. The solid line in Figure 4d shows the best fitting curve and a good agreement is reached between the data and the model with the fitting parameters $\Gamma_{0}=(23 \pm 1) \mathrm{meV}, \gamma_{\mathrm{ac}}=(30 \pm 5) \mu \mathrm{eV} \mathrm{K} \mathrm{K}^{-1}$, $\gamma_{\mathrm{LO}}=(75 \pm 5) \mathrm{meV}, E_{\mathrm{LO}}=(19 \pm 1) \mathrm{meV}$. These values well agree with data in the literature. ${ }^{[32,39]}$

A further evidence proving that the emission band at low temperature in ETL 4 must be attributed to a tetragonal crystalline phase of MAPI with good quality, is given in Figure 5a. We report two spectra, already shown before, from side A (red curve) and side B (black curve), acquired at the same average excitation intensity $I_{0}=10 \mathrm{~W} \mathrm{~cm}{ }^{-2}$. Decreasing the excitation intensity by one order of magnitude, the emission from side $\mathrm{B}$ changes completely, showing a spectrum (blue curve) similar to the side A (red curve), with the dominant contribution of the traps and a small signal from the orthorhombic phase. This is explained by the fact that, lowering the power density, only the 

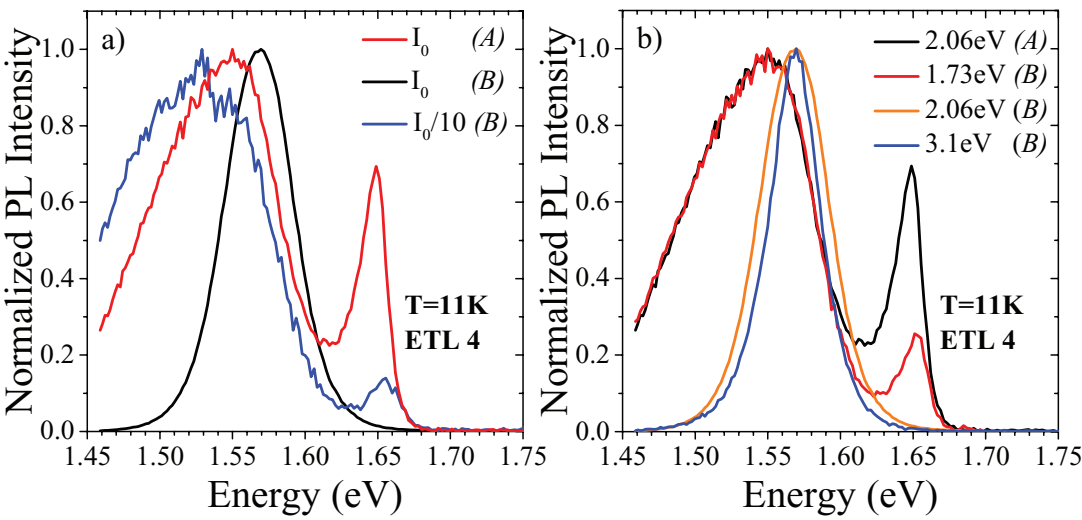

Figure 5. a) Normalized PL spectra of ETL 4 at $T=11 \mathrm{~K}$, after excitation at $2.06 \mathrm{eV}$, for different excitation densities and excitation sides (in brackets). $I_{0}$ corresponds to an average intensity of $10 \mathrm{~W} \mathrm{~cm}^{-2}$. b) Normalized PL spectra of ETL 4 at $T=11 \mathrm{~K}$ for different excitation photon energies and excitation sides (in brackets).

trap energy levels close to the band gap tail are filled. In addition, the PL spectrum of the sample ETL 4, obtained by exciting from side B with an intensity of $I_{0} / 10$ (blue curve in Figure 5a), is very similar to the spectra of the ETLs 1, 2, and 3 (Figure 3b) by exciting from side B with a higher intensity $I_{0}$. This means that the trap density in the mesoporous region of MAPI in ETL 4 is lower, of about one order of magnitude, with respect to the other samples. This result agrees with the better crystal quality found by SEM analysis for ETL 4 (see Figure S4 in the Supporting Information).

As stated previously, to confirm that the crystalline nature of the film changes when in contact with the ETL, in particular in presence of GO-Li plus $\mathrm{G}+\mathrm{mTiO}_{2}$, we probed the ETL 4 along the thickness exploiting the different absorption coefficient of MAPI varying the excitation photon energies. We spanned the range from 1.73 to $3.1 \mathrm{eV}$, where FTO and $\mathrm{cTiO}_{2}$ have a low and nearly constant absorption. The result of this experiment, performed at $11 \mathrm{~K}$, is reported in Figure $5 \mathrm{~b}$. Let us focus on side B. At high photon energy excitation, $3.1 \mathrm{eV}$ (blue curve) and $2.06 \mathrm{eV}$ (orange curve), the MAPI is excited for a few tens of nanometers close to the ETL and, as already observed above, the spectra show only the tetragonal phase (which should not be observed at this temperature since the only stable phase below $150 \mathrm{~K}$ is the orthorhombic). Decreasing the excitation photon energy down to $1.73 \mathrm{eV}$, the absorption coefficient of MAPI decreases and therefore the sample is excited more uniformly in depth. The resulting spectrum (red curve) shows an increase of the contribution of the orthorhombic phase. For comparison, a spectrum from side A with an excitation of $2.06 \mathrm{eV}$ is reported (black curve). The comparison of this spectrum with that at $1.73 \mathrm{eV}$ from side B (red curve) shows, apart from a difference in the intensity of the orthorhombic phase, exactly the same contribution from the radiative traps and the tetragonal phase. The observed behavior proves that the crystalline nature of MAPI at low temperature is influenced by the interaction with the ETL, which inhibits the MAPI phase change into the orthorhombic form.

Our results demonstrate a substantial improvement of the active layer morphology of ETL 4 with an efficient carrier capture from the ETL. This is confirmed by a remarkable increase in power conversion efficiency for complete devices, obtained by $I-V$ characterization (see Figure S6, S7 and Table S1 in the Supporting Information), which is mainly ascribed to an improved $J_{\mathrm{sc}}$.

In order to exclude that the increase of the $J_{\mathrm{sc}}$ of the solar cells with graphenebased ETLs were merely due to an increase of the absorption in the active layer, the absorbance spectra of all our photoelectrodes were measured (see Figure S8 of the Supporting Information). The absorbance spectra are all very similar with a slightly lower absorbance for graphenebased ETLs. Therefore the contribution of the absorption to the increase of the $J_{\mathrm{sc}}$ must be excluded, proving that it stems from an improved electron charge transfer from perovskite to graphene-based ETL. As a further confirmation of our conclusions, open-circuit photovoltage decay measurements were performed, as reported in Figure S9 of the Supporting Information. They showed a remarkable decrease in charge recombination within the graphene-based ETL and therefore a lower concentration of detrimental defects, thus justifying the improved charge collection when ETL 4 is used in the complete device.

\section{Conclusions}

We investigated the effects of different graphene-based ETLs in sensitized MAPI photoelectrodes. In particular we have compared four different samples with the following ETLs: $\mathrm{mTiO}_{2}$, $\mathrm{G}+\mathrm{mTiO}_{2}, \mathrm{mTiO}_{2}$ plus GO-Li interlayer, and $\mathrm{G}+\mathrm{mTiO}_{2}$ plus GO-Li interlayer. We have studied the ETL effects on the carrier collection efficiency and on the MAPI morphology and quality along the thickness. In presence of ETL, we found faster PL decays by exciting on FTO side with respect to the MAPI side which is explained by efficient electron removal from MAPI layer due to the ETL. In particular an increase of the electron collection efficiency up to a factor 3 with respect to standard $\mathrm{mTiO}_{2}$ is reported.

Moreover, the MAPI layer embedded in $\mathrm{G}+\mathrm{mTiO}_{2}$ plus GO-Li ETL shows a crystalline quality much better than the other samples, with a trap density about one order of magnitude lower. Exploiting the dispersion of the MAPI absorption coefficient, we could probe the sample along the thickness, finding that the morphology of the MAPI film embedded in the $\mathrm{G}+\mathrm{mTiO}_{2}$ plus GO-Li ETL is frozen in the tetragonal phase, regardless of the temperature. Moreover, the observed morphology improvement of the MAPI encapsulated in the $\mathrm{mTiO}_{2}$ plus GO-Li layer supports the increased efficiency measured for the complete devices. Finally, our results show that graphene based ETLs significantly improve both the carrier collection and the crystalline quality of the active material, opening new routes to the development of efficient and stable MAPI solar cells. 


\section{Experimental Section}

Sample Preparation: Solar cells photoelectrodes were prepared on FTO conductive glass (Pilkington TEC $8,8 \Omega \square^{-1}, 25 \mathrm{~mm} \times 25 \mathrm{~mm}$ ). The substrates were cleaned in an ultrasonic bath, using three sequential steps: detergent with deionized water, acetone, and 2-propanol (10 min for each step). The substrates were covered by a compact layer of $\mathrm{TiO}_{2}$ $\left(\mathrm{cTiO}_{2}\right)$. A solution of acetylacetone $(2 \mathrm{~mL})$, titanium diisopropoxide $(3 \mathrm{~mL})$, and ethanol $(45 \mathrm{~mL})$ was deposited onto the FTO substrates by Spray Pyrolysis Deposition at $450{ }^{\circ} \mathrm{C}$. The final thickness of the $\mathrm{CTiO}_{2}$ layer was measured about $50 \mathrm{~nm}$ by a Dektak Veeco 150 profilometer.

The $\mathrm{mTiO}_{2}$ layer was obtained starting by an ethanol solution of 18NR-T titania paste (Dyesol) dissolved in pure ethanol (1:5 by weight), stirred overnight. The graphene-doped $\mathrm{mTiO}_{2}$ was obtained by adding sonicated graphene ink (1\% in volume), prepared by dispersing $5 \mathrm{~g}$ of graphite flakes (+100 mesh, $\geq 75 \%$, Sigma Aldrich) in $500 \mathrm{~mL}$ of $\mathrm{N}$-methyl-2-pyrrolidone (NMP) (Sigma Aldrich). The initial dispersion was ultrasonicated (VWR) for $6 \mathrm{~h}$ and subsequently ultracentrifuged using a SW32Ti rotor in a Beckman-Coulter Optima XPN ultracentrifuge at $10000 \mathrm{rpm}(\approx 12200 \mathrm{~g})$ for $30 \mathrm{~min}$ at $15^{\circ} \mathrm{C}$. After ultracentrifugation, the upper $80 \%$ supernatant was extracted by pipetting. The concentration of the graphitic flakes is calculated from the optical absorption spectroscopy (OAS) (see Figure S1 in the Supporting Information), giving a concentration of $0.25 \mathrm{~g} \mathrm{~L}^{-1}$. The morphology of the flakes, i.e., lateral size and thicknesses were characterized by TEM and AFM, respectively (see Figure S2 in the Supporting Information) giving a lateral size distribution of $150 \mathrm{~nm}$ and thickness of $1.7 \mathrm{~nm}$. Raman spectroscopy data are found in Figure $\mathrm{S} 3$ in the Supporting Information. Both standard $\mathrm{mTiO}_{2}$ and $\mathrm{G}+\mathrm{mTiO}_{2}$ dispersions were sonicated $10 \mathrm{~min}$ prior to be spin-coated in air at $1700 \mathrm{rpm}$ for $20 \mathrm{~s}$ onto the $\mathrm{CTiO}_{2}$ surface, followed by a calcination step at $450{ }^{\circ} \mathrm{C}$ for $30 \mathrm{~min}$.

The GO-Li interlayer was realized by spin coating (2000 rpm for $10 \mathrm{~s}$ ) $200 \mu \mathrm{L}$ of $\mathrm{GO}-\mathrm{Li}$ dispersion in ethanol $/ \mathrm{H}_{2} \mathrm{O}(3: 1)$ prepared as reported in ref. [40]. After the deposition, the substrates were annealed at $110^{\circ} \mathrm{C}$ for $10 \mathrm{~min}$.

The photoelectrodes were completed by depositing the perovskite active layer in dry conditions (relative humidity less than $30 \%$ ) by a double step method: a lead iodide solution $\mathrm{Pbl}_{2}$ in $\mathrm{N}, \mathrm{N}$-dimethylformamide, $1 \mathrm{M}$, heated at $70{ }^{\circ} \mathrm{C}$ ) was spin coated at $6000 \mathrm{rpm}$ for $10 \mathrm{~s}$ on heated substrates $\left(50{ }^{\circ} \mathrm{C}\right)$ which were then dipped into a $\mathrm{CH}_{3} \mathrm{NH}_{3} \mathrm{I}$ (Dyesol) in anhydrous 2-propanol solution $\left(10 \mathrm{mg} \mathrm{mL}^{-1}\right)$ for $15 \mathrm{~min}$. Finally, the samples were heated at $80{ }^{\circ} \mathrm{C}$ for $20 \mathrm{~min}$ in air. The MAPI absorbing layer has a typical thickness of $350 \mathrm{~nm}$ with a perovskite penetration into mesoporous layer of about $200 \mathrm{~nm}$. The samples were not encapsulated. Complete solar cells were realized with the same structure of the investigated samples to compare the $\mathrm{PL}$ results with the current-voltage (I-V) characteristics. In the case of complete devices doped spiroOMeTAD (73.5 $\mathrm{mg} \mathrm{mL}^{-1}$ ) in chlorobenzene solution doped with tertbutylpyridine (TBP $26.77 \mu \mathrm{L} \mathrm{mL}^{-1}$ ), lithium bis(trifluoromethanesulfonyl) imide (LiTFSI $16.6 \mu \mathrm{L} \mathrm{mL} \mathrm{m}^{-1}$ ), and cobalt(III) complex (FK209 from Lumtec, $7.2 \mu \mathrm{L} \mathrm{mL}^{-1}$ ) was spin coated (2000 rpm for $20 \mathrm{~s}$ ) onto the tested photoelectrodes. The final devices were completed by Au counter-electrode thermal evaporation (100 nm). I-V characteristics were recorded under AM1.5G solar simulator Solar Constant from KHS at $1000 \mathrm{~W} \mathrm{~m}^{-2}$ (1 sun). Results on the solar cells are shown in the Supporting Information.

Optical Absorption Spectroscopy: The OAS of the as-produced inks was carried out in the $300-1000 \mathrm{~nm}$ range with a Cary Varian 5000 UV-Vis-NIR spectrometer. The absorption spectra were acquired using a $1 \mathrm{~mL}$ quartz glass cuvette. The ink was diluted to 1:7 in NMP. The NMP solvent baseline was subtracted. The concentration of graphitic flakes was determined from the extinction coefficient at $660 \mathrm{~nm}$, using $A=\alpha / c$, where $/[\mathrm{m}]$ is the light path length, $c\left[\mathrm{~g} \mathrm{~L}^{-1}\right]$ is the concentration of dispersed graphitic material, and $\alpha\left[\mathrm{L} \mathrm{g}^{-1} \mathrm{~m}^{-1}\right]$ is the absorption coefficient, with $\alpha \sim 1390 \mathrm{~L} \mathrm{~g}^{-1} \mathrm{~m}^{-1}$ at $660 \mathrm{~nm}$. ${ }^{[1]}$

Transmission Electron Microscopy: The exfoliated flakes morphology was characterized by using a TEM JOEL JEM 1011, using an acceleration voltage of $100 \mathrm{kV}$. The sample preparation was performed diluting the ink in NMP (1:10). $20 \mu \mathrm{L}$ of the diluted sample were drop cast on copper grids (200 mesh), and dried in vacuum overnight. Statistical analyses were fitted with log-normal distributions.

Atomic Force Microscopy: The dispersions were diluted 1:30 in NMP. $100 \mu \mathrm{L}$ of the dilutions were drop-casted onto $\mathrm{Si} / \mathrm{SiO}_{2}$ wafers. AFM images were acquired with Bruker Innova AFM in tapping mode using silicon probes (frequency $=300 \mathrm{kHz}$, spring constant $=40 \mathrm{~N} \mathrm{~m}^{-1}$ ). Statistical analysis were fitted with log-normal distributions.

Raman Spectroscopy: The graphene inks were drop-cast onto Si/ $\mathrm{SiO}_{2}$ wafers (LDB Technologies Ltd.) and dried under vacuum. Raman measurements were collected with a Renishaw inVia confocal Raman microscope using an excitation line of $514 \mathrm{~nm}$ with a 100x objective lens, and an incident power of $\approx 1 \mathrm{~mW}$ on the sample. 20 spectra were collected for each sample. Peaks were fitted with Lorentzian functions.

Scanning Electron Microscopy: Electrodes were imaged by aim of a field-emission SEM (JOEL JSM-7500 FA). The acceleration voltage was set at $5 \mathrm{kV}$. Images were collected using the in-lens sensors (secondary electron in-lens image) and the secondary electron sensor (lower secondary electron image). No coating was applied.

Photoluminescence Spectroscopy: PL experiments were performed, in a quasi back-scattering geometry, keeping the samples in a closed cycle cryostat and the temperature was changed from 10 to $300 \mathrm{~K}$. TI-PL measurements were performed exciting the samples by different modelocked ps laser sources: a tunable (700-850 and 350-425 nm with the second harmonic generator) Ti-Sapphire laser operating at $81.3 \mathrm{MHz}$ repetition rate with 1.2 ps pulses and a 4 ps Rhodamine 6G dye laser synchronously pumped by the second harmonic of a mode-locked Nd-YAG laser, operating at $76 \mathrm{MHz}$. The $\mathrm{PL}$ signal was spectrally dispersed by a $50 \mathrm{~cm}$ monochromator providing a spectral resolution of $1 \mathrm{meV}$ and detected by a microchannel plate photomultiplier. TR-PL measurements were carried out exciting the samples by the ps dye laser operating at $600 \mathrm{~nm}$ and using TCSPC with a temporal resolution of about 60 ps.

\section{Supporting Information}

Supporting Information is available from the Wiley Online Library or from the author.

\section{Acknowledgements}

F.B. acknowledges funding from the Italian Ministry for Education, University and Research within the Futuro in Ricerca (FIRB) program (project DeLIGHTeD, Protocollo RBFR12RS1W). This work was partially supported by ENTE CARIFI grant no. 2015/11162 and from the European Union's Horizon 2020 research and innovation programme under grant agreement no. 696656 - GrapheneCore1. All the authors warmly acknowledge Franco Bogani for fruitful discussions.

\section{Conflict of Interest}

The authors declare no conflict of interest.

\section{Keywords}

electron transport layer, graphene, perovskite solar cells, photoluminescence 
[1] M. Grätzel, Nat. Mater. 2014, 13, 838.

[2] G. Hodes, Science 2013, 342, 317.

[3] Z. Xiao, Y. Yuan, Q. Wang, Y. Shao, Y. Bai, Y. Deng, Q. Dong, M. Hu, C. Bi, J. Huang, Mater. Sci. Eng., R 2016, 101, 1.

[4] M. M. Lee, J. Teuscher, T. Miyasaka, T. N. Murakami, H. J. Snaith, Science 2012, 338, 643.

[5] J. H. Heo, S. H. Im, J. H. Noh, T. N. Mandal, C.-S. Lim, J. A. Chang, Y. H. Lee, H.-j. Kim, A. Sarkar, M. K. Nazeeruddin, M. Grätzel, S. I. Seok, Nat. Photonics 2013, 7, 486.

[6] J. You, Z. Hong, Y. M. Yang, Q. Chen, M. Cai, T.-B. Song, C.-C. Chen, S. Lu, Y. Liu, H. Zhou, Y. Yang, ACS Nano 2014, 8, 1674.

[7] H. Zhou, Q. Chen, G. Li, S. Luo, T.-b. Song, H.-S. Duan, Z. Hong, J. You, Y. Liu, Y. Yang, Science 2014, 345, 542.

[8] F. Nanni, F. R. Lamastra, F. Franceschetti, F. Biccari, I. Cacciotti, Ceram. Int. 2014, 40, 1851.

[9] A. Kojima, K. Teshima, Y. Shirai, T. Miyasaka, J. Am. Chem. Soc. 2009, 131, 6050 .

[10] NREL Best Research-Cell Efficiency Chart, https://www.nrel.gov/pv/ (accessed: November 2016).

[11] S. A. Veldhuis, P. P. Boix, N. Yantara, M. Li, T. C. Sum, N. Mathews, S. G. Mhaisalkar, Adv. Mater. 2016, 28, 6804

[12] B. R. Sutherland, E. H. Sargent, Nat. Photonics 2016, 10, 295.

[13] A. L. Palma, L. Cinà, Y. Busby, A. Marsella, A. Agresti, S. Pescetelli, J.-J. Pireaux, A. Di Carlo, ACS Appl. Mater. Interfaces 2016, 8, 26989.

[14] A. L. Palma, L. Cinà, Y. Busby, A. Marsella, A. Agresti, S. Pescetelli, J.-J. Pireaux, A. Di Carlo, Phys. Status Solidi 2016, 13, 958.

[15] T. Xu, L. Chen, Z. Guo, T. Ma, Phys. Chem. Chem. Phys. 2016, 18, 27026.

[16] S. Draguta, S. Thakur, Y. V. Morozov, Y. Wang, J. S. Manser, P. V. Kamat, M. Kuno, J. Phys. Chem. Lett. 2016, 7, 715.

[17] X. Wu, M. T. Trinh, D. Niesner, H. Zhu, Z. Norman, J. S. Owen, O. Yaffe, B. J. Kudisch, X.-Y. Zhu, J. Am. Chem. Soc. 2015, 137, 2089.

[18] J. Kim, S.-H. Lee, J. H. Lee, K.-H. Hong, J. Phys. Chem. Lett. 2014, 5,1312

[19] Organic-Inorganic Halide Perovskite Photovoltaics (Eds: N.-G. Park, M. Grätzel, T. Miyasaka), Springer Nature, Switzerland 2016.

[20] D.-Y. Son, J.-W. Lee, Y. J. Choi, I.-H. Jang, S. Lee, P. J. Yoo, H. Shin, N. Ahn, M. Choi, D. Kim, N.-G. Park, Nat. Energy 2016, 1, 16081.

[21] F. Giordano, A. Abate, J. P. C. Baena, M. Saliba, T. Matsui, S. H. Im, S. M. Zakeeruddin, M. K. Nazeeruddin, A. Hagfeldt, M. Grätzel, Nat. Commun. 2016, 7, 10379.

[22] D. W. deQuilettes, W. Zhang, V. M. Burlakov, D. J. Graham, T. Leijtens, A. Osherov, V. Bulović, H. J. Snaith, D. S. Ginger, S. D. Stranks, Nat. Commun. 2016, 7, 11683.

[23] Y. Shao, Y. Yuan, J. Huang, Nat. Energy 2016, 1, 15001.
[24] S. Y. Leblebici, L. Leppert, Y. Li, S. E. Reyes-Lillo, S. Wickenburg, E. Wong, J. Lee, M. Melli, D. Ziegler, D. K. Angell, D. F. Ogletree, P. D. Ashby, F. M. Toma, J. B. Neaton, I. D. Sharp, A. Weber-Bargioni, Nat. Energy 2016, 1, 16093.

[25] M. Acik, S. B. Darling, J. Mater. Chem. A 2016, 4, 6185.

[26] A. Agresti, S. Pescetelli, B. Taheri, A. E. Del Rio Castillo, L. Cinà, F. Bonaccorso, A. Di Carlo, ChemSusChem 2016, 9, 2609.

[27] A. Capasso, F. Matteocci, L. Najafi, M. Prato, J. Buha, L. Cinà, V. Pellegrini, A. D. Carlo, F. Bonaccorso, Adv. Energy Mater. 2016, 6, 1600920.

[28] N. Ahn, K. Kwak, M. S. Jang, H. Yoon, B. Yang, J. Lee, P. V. Pikhitsa, J. Byun, M. Choi, Nat. Commun. 2016, 7, 13422.

[29] A. Agresti, S. Pescetelli, A. L. Palma, A. E. D. R. Castillo, D. Konios, G. Kakavelakis, S. Razza, L. Cinà, E. Kymakis, F. Bonaccorso, A. D. Carlo, ACS Energy Lett. 2017, 2, 279.

[30] P. Löper, M. Stuckelberger, B. Niesen, J. Werner, M. Filipič, S.-J. Moon, J.-H. Yum, M. Topič, S. D. Wolf, C. Ballif, J. Phys. Chem. Lett. 2015, 6, 66

[31] R. L. Milot, G. E. Eperon, H. J. Snaith, M. B. Johnston, L. M. Herz, Adv. Funct. Mater. 2015, 25, 6218

[32] M. I. Dar, G. Jacopin, S. Meloni, A. Mattoni, N. Arora, A. Boziki, S. M. Zakeeruddin, U. Rothlisberger, M. G. tzel, Sci. Adv. 2016, 2, e1601156.

[33] Y. Bi, E. M. Hutter, Y. Fang, Q. Dong, J. Huang, T. J. Savenije, J. Phys. Chem. Lett. 2016, 7, 923.

[34] S. C. Warren, A. Margineanu, D. Alibhai, D. J. Kelly, C. Talbot, Y. Alexandrov, I. Munro, M. Katan, C. Dunsby, P. M. W. French, PLOS ONE 2013, 8, e70687.

[35] K. Pydzińska, J. Karolczak, I. Kosta, R. Tena-Zaera, A. Todinova, J. Idígoras, J. A. Anta, M. Ziółek, ChemSusChem 2016, 9, 1647.

[36] W. Kong, Z. Ye, Z. Qi, B. Zhang, M. Wang, A. Rahimi-Iman, H. Wu, Phys. Chem. Chem. Phys. 2015, 17, 16405.

[37] H.-H. Fang, R. Raissa, M. Abdu-Aguye, S. Adjokatse, G. R. Blake, J. Even, M. A. Loi, Adv. Funct. Mater. 2015, 25, 2378.

[38] A. Dobrovolsky, A. Merdasa, E. L. Unger, A. Yartsev, I. G. Scheblykin, Nat. Commun. 2017, 8, 34.

[39] A. D. Wright, C. Verdi, R. L. Milot, G. E. Eperon, M. A. Pérez-Osorio, H. J. Snaith, F. Giustino, M. B. Johnston, L. M. Herz, Nat. Commun. 2016, 7, 11755.

[40] A. Agresti, S. Pescetelli, L. Cinà, D. Konios, G. Kakavelakis, E. Kymakis, A. Di Carlo, Adv. Funct. Mater. 2016, 26, 2686.

[41] M. Lotya, Y. Hernandez, P. J. King, R. J. Smith, V. Nicolosi, L. S. Karlsson, F. M. Blighe, S. D. Zhiming, L. T. McGovern, G. S. Duesberg, J. N. Coleman, J. Am. Chem Soc. 2009, 131, 3611. 